\title{
CCD PHOTOMETRIC AND SPECTROSCOPIC OBSERVATIONS OF THE YOUNG OPEN CLUSTER NGC 433
}

\author{
P. Battinelli \\ Osservatorio Astronomico di Roma, viale del Parco Mellini 84, I-00136, Roma, Italy \\ R. Capuzzo-Dolcetta and R. Nesci \\ Istituto Astronomico Università 'La Sapienza' via G. M. Lancisi 29, I-00161, Roma, Italy \\ Received 15 October 1991; revised 19 December 1991
}

\begin{abstract}
In this paper we present the first CMD diagram and spectral types of stars in the field of NGC 433. The cluster is rather poor, containing 12 stars down to $V=16$ in a radius of 106 arcsec around R.A. $1^{\mathrm{h}} 15^{\mathrm{m}}$ $07^{\mathrm{s}}$; Dec. $+60^{\circ} 11^{\prime} 11^{\prime \prime} .7(2000.0)$. The averaged reddening is $\langle\mathrm{E}(B-V)\rangle=0.8$. The brightest stars are B5, corresponding to an age $<110 \mathrm{Myr}$. The derived cluster distance of $2.1 \mathrm{Kpc}$ is compatible with a physical association with an $\mathrm{H}$ I cloud detected in the cluster direction, while the $\mathrm{CO}$ clouds observed in the same direction are very likely in the foreground. Within the limits of our photometry, the cluster consists of a clump of B stars and is lacking of fainter stars. This work supports the idea that the population of disk stellar clusters cannot be sharply divided into "true" bound open clusters and unbound stellar groups.
\end{abstract}

\section{INTRODUCTION}

Recently, the CO survey by Leisawitz et al. (1989) has given a large deal of data for molecular clouds around young open clusters, which is very useful to study the interaction between gas and stars in regions of recent star formation. The reliability of the conclusion drawn on the stellar-and gaseous-components interaction stands on the possibility to state the physical connection between molecular cloud and cluster, which requires a good determination of the cluster distance. Moreover, some of the surveyed young clusters are very loose and poor, making it even difficult to define them as physical systems and not mere fluctuations on the background. This is the case of NGC 433 [R.A. $1^{\mathrm{h}} 15^{\mathrm{m}} 07^{\mathrm{s}}$; Dec. $\left.+60^{\circ} 11^{\prime} 11^{\prime \prime} .7(2000.0) ; l=125.9, b=-2.6\right]$, which overlaps on the sky with a CO cloud having a radial velocity $V_{\text {LSR }}$ (with respect to the local standard of rest) between -9 and $-12 \mathrm{~km} \mathrm{~s}^{-1}$ and with an $\mathrm{H}$ I cloud complex with $V_{\text {LSR }}$ between -17.5 and $-23.7 \mathrm{~km} \mathrm{~s}^{-1}$ (Leisawitz \& De Geus 1991). The cluster is classified in the Alter et al. (1970) catalogue as a small OB cluster. In the POSS prints it is hardly even recognizable as a cluster, in what appears like a poor clump of stars around the bright BD + 59217 star. The only available photometric observations of this cluster date back to Alter (1944), who gave photographic magnitudes obtained with the Mond Astrograph of the Norman Lockyer Observatory for 14 stars whose membership was evaluated on the basis of their apparent distance (see Alter 1940). The NGC 433 estimated distance was $4.5 \mathrm{kpc}$, corresponding to a $V_{\mathrm{LSR}}$ of about $-50 \mathrm{~km} \mathrm{~s}^{-1}$.

The previous considerations prompted us to perform photometric observations of NGC 433, in order to check, first, if the clump of stars is statistically consistent with the existence of a 'true' stellar cluster, and-in this case - to deduce more reliable estimates of its distance, size, and age.

\section{PHOTOMETRIC OBSERVATIONS}

The identification of a loose cluster on a crowded background is best obtained using a wide field instrument, which allows us to map the star counts on a region of the sky much larger than the expected cluster radius. With the introduction of the CCD detectors, the average field of view of the telescopes has dropped to a few arcminutes. In order to have both the advantages of a large field and of a CCD detector, we choose to perform our photometric observations of NGC 433 with the $60 / 90 / 180 \mathrm{~cm}$ Schmidt telescope of the Rome Astronomical Observatory at Campo Imperatore. The telescope allows observations of a $17 \times 25$ arcmin field on a nitrogen-cooled CCD camera of $384 \times 576$ pixels ( 2.64 arcsec/pixel) (Natali \& Pedichini 1990) and standard Johnson's filters.

To prevent pixel saturation from the brightest stars (a range of 3.5 mag may be covered by a single exposure), we summed up a few short exposures to obtain long exposure frames. The integration time ranged from 60 to $720 \mathrm{~s}$. Flatfielding was performed on the twilight sky, covering the telescope mouth with a white sheet as a light diffusor. For calibration purposes, we observed in the same night the cluster NGC 436, which is very near to NGC 433 on the sky $\left(1.3^{\circ} \mathrm{S}\right)$ and was recently photoelectrically observed in the Johnson's system by Huestamendia et al. (1991).

All the data reduction was performed with homemade software. Given the small scale of the telescope, no attempt was made to fit the stellar light distribution with an analytical Point Spread Function (PSF), so instrumental magnitudes were derived by a simple aperture photometry. The photometric radius was set at 2 pixels after some trials, in order to include as much star light as possible and prevent contamination from nearby objects.

Calibration constants were derived for each band from $\approx 30$ stars belonging to NGC 436 . The relations between instrumental and photoelectric $B$ and $V$ magnitudes have been found linear; as shown by Figs. 1(b), 1(c) the rms scatter from the 1:1 relation is 0.085 and 0.066 for $B$ and $V$, respectively. Figure 1 (a) shows a significant nonlinearity of the correlation between instrumental and photoelectric $U$ magnitudes. Taking into account that a reliable color correction cannot be evaluated from the NGC 436 sample (which 

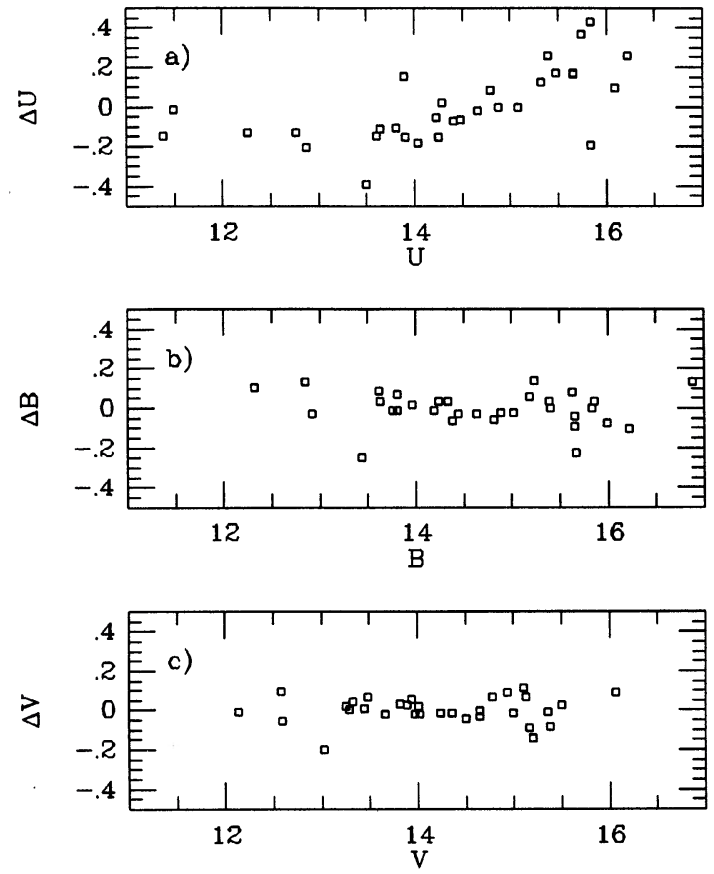

FIG. 1. (a)-(c) Differences between our instrumental and Huestamendia et al. (1991) photoelectric magnitudes of NGC 436 stars vs instrumental magnitude. Panels $a, b, c$ refer to $U, B, V$ filters, respectively.

lacks of red stars) and that the dispersion of $U$ data is much larger than for $B$ and $V$, we decided not to use our $U$ observations.

\subsection{The Cluster Definition and Color-Magnitude Diagram}

In his pioneering paper, Alter (1944) estimated, for NGC 433 , a radius of 3.4 arcmin, corresponding to 77 pixel on our frames. Only 14 stars down to the 14 th photographic magnitude are listed by him as cluster members: no finding chart is given, but only coordinates as offsets with 0.1 arcmin accuracy from the bright star HD 7332 (BD + 59211 ).

To better assess the cluster richness, we scanned first with an automatic star-finding routine the best exposed $V$ frame down to $V \approx 16$. The star list so obtained was then used for all the frames to perform the aperture photometry of each star, centered on the pixel nearest to the star light distribution center. A few blended stars near the cluster center were later identified and measured interactively with the aperture centered on the brightest pixel. A total of 369 stars were found, giving an average of 3140 stars per square degree.

Starting from the center given by Alter (1944), we computed the star density in concentric rings for some limiting magnitudes, and the results are given in Fig. 2. The background level is reached at different distances from the center by varying the cutoff magnitude, decreasing from $\approx 80$ pixels $(211$ arcsec) for the deepest sample to $\approx 20$ pixels ( 53 arcsec) for the brightest one. To improve the cluster radius determination, we redefined the cluster center as the barycenter of stars brighter than $V=15$ in a circular area of 50 pixels ( 132 arcsec) radius around the center given by Alter (1944) and computed again the star density profile. We chose $V=15$ instead of $V=16$ because it is logical to expect a young cluster better emerging from the background at brighter magnitudes. Figure 3 shows that the peak densities so obtained are higher than those derived with the previous center, making us confident of the reliability of this new center, whose coordinates are R.A. $1^{\mathrm{h}} 15^{\mathrm{m}} 07^{\mathrm{s}}$; Dec. $+60^{\circ} 11^{\prime}$ $11^{\prime \prime} .7(2000.0)$, i.e., $42^{\prime \prime}$ south and $1^{\text {s }}$ west of BD +59217. There is, furthermore, a decrease of the ratio of the peak density to the background level at decreasing cutoff luminosity, as expected for an open cluster, whose stellar population is, on the average, younger than the background one. The background level is reached at $\approx 40$ pixels ( 106 arcsec) from the center for any cutoff magnitude, and we take this value as an estimate of the cluster size. On the basis of the back-
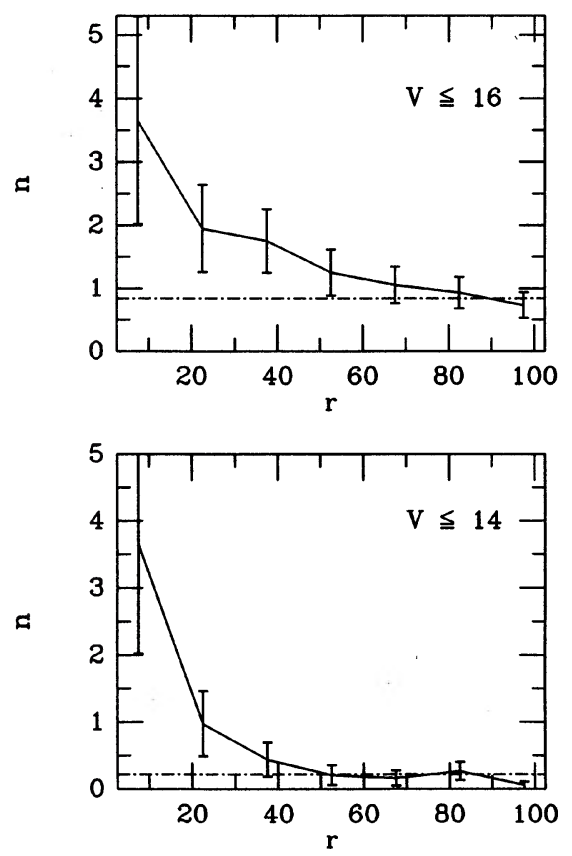
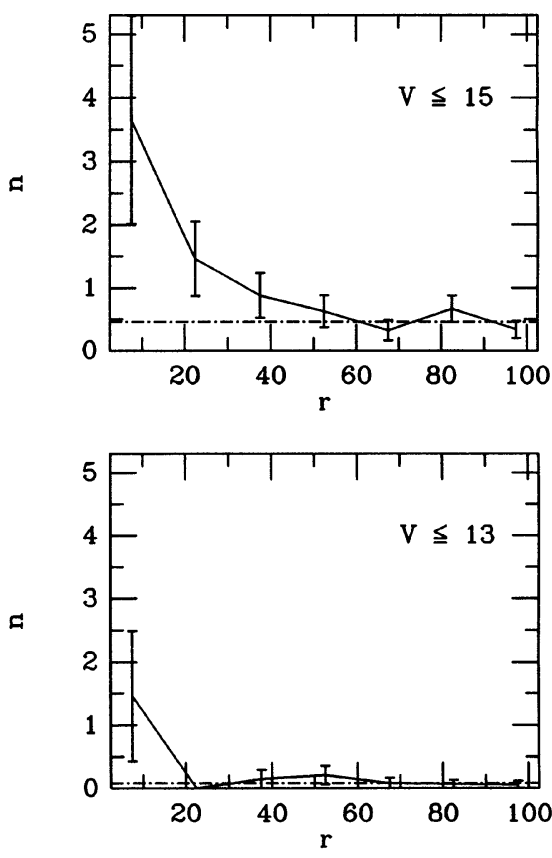

FIG. 2. Star count profiles (in arc$\min ^{-2}$ ) in function of the distance (in pixels, 1 pixel $\left.=2.64^{\prime \prime}\right)$ from the center assumed by Alter (1944). Each panel is labeled by the cutoff $V$ magnitude. The error bars are the $\sqrt{N}$ fluctuations. 

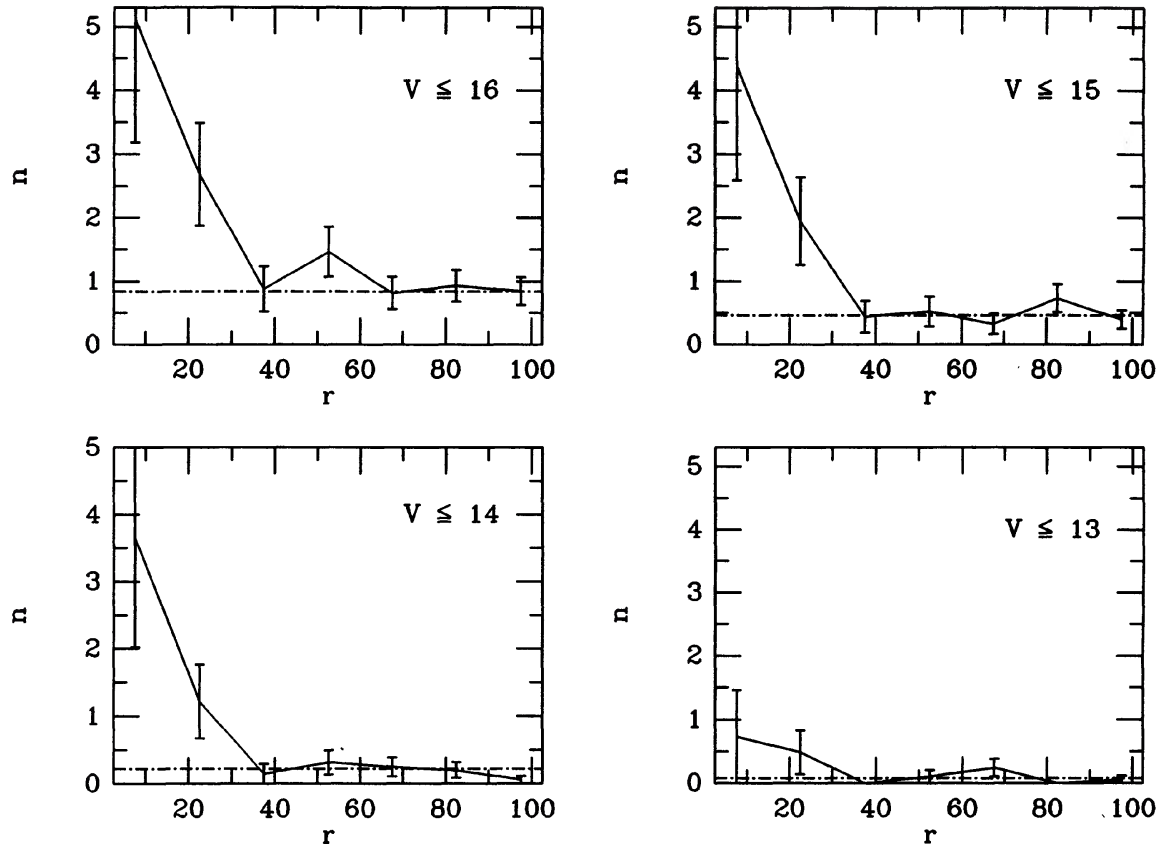

Fig. 3. As Fig. 2, but with cluster center as defined in Sec. 2.1 .

ground star density, we expect only 8 stars, out of a total of 20 down to $V=16$ detected in this area, to be field stars. So, we can consider this radius as a "fiducial" cluster radius. We report in Table 1 the $B$ and $V$ magnitudes of all the 47 stars within 185 arcsec ( 70 pixels) from the cluster center-i.e., an area somewhat larger than the previously defined onetogether with their coordinates in pixels. Figure 4 is the find- ing chart. The color-magnitude diagram of the stars listed in Table 1 is given in Fig. 5 .

The continuous line shown in Fig. 5 is the Zero Age Main Sequence (ZAMS) taken from Schmidt-Kaler (1982) corrected for $\mathrm{E}(B-V)=0.8$ and an apparent distance modulus of 14.1 , corresponding to $2100 \mathrm{pc}$ (see Sec. 3 ). Note that the Schmidt-Kaler's compilation does not give $(B-V)_{0}$

TABLE 1. Coordinates (in pixels, 1 pixel $=2.64$ arcsec) and magnitudes of measured stars within 185 arcsec from the center of NGC 433 (those inside 106 $\operatorname{arcsec}$ are identified by $*$ ). Right ascension increases with decreasing $x$; declination increases with increasing $y$. Cluster center is at $x=150, y=265$. In brackets Alter's (1944) identification numbers.

\begin{tabular}{|c|c|c|c|c|c|c|c|c|c|}
\hline Star & $x$ & $y$ & $B$ & $V$ & Star & $x$ & $y$ & $B$ & $V$ \\
\hline $\begin{array}{l}1 \\
2 \\
3 \\
4 \\
5 \\
6 \\
7 \\
8 \\
9 \\
10 \\
11^{*} \\
12 \\
13 \\
14 \\
15 \\
16^{*} \\
17^{*} \\
18^{*}(12) \\
19^{*} \\
20^{*} \\
21^{*}(11) \\
22^{*} \\
23^{*}(18) \\
24^{*}(15)\end{array}$ & $\begin{array}{l}185 \\
167 \\
169 \\
144 \\
102 \\
166 \\
174 \\
195 \\
138 \\
120 \\
168 \\
114 \\
205 \\
199 \\
116 \\
131 \\
135 \\
157 \\
161 \\
135 \\
153 \\
142 \\
179 \\
164\end{array}$ & $\begin{array}{l}210 \\
209 \\
216 \\
219 \\
222 \\
220 \\
226 \\
223 \\
226 \\
229 \\
232 \\
234 \\
234 \\
239 \\
243 \\
247 \\
250 \\
249 \\
251 \\
250 \\
254 \\
257 \\
260 \\
261\end{array}$ & $\begin{array}{l}11.30 \\
14.20 \\
16.50 \\
16.16 \\
12.09 \\
15.83 \\
11.35 \\
16.15 \\
16.12 \\
16.56 \\
15.53 \\
15.64 \\
15.90 \\
16.02 \\
15.95 \\
15.10 \\
12.54 \\
15.15 \\
15.76 \\
14.12: \\
14.27 \\
16.31 \\
15.84 \\
16.03\end{array}$ & $\begin{array}{l}10.98 \\
13.80 \\
15.72 \\
15.44 \\
11.62 \\
15.20 \\
11.04 \\
14.98 \\
15.39 \\
15.47 \\
14.47 \\
14.78 \\
14.81 \\
15.36 \\
15.28 \\
14.42 \\
12.09 \\
14.56 \\
15.72 \\
13.62: \\
13.68 \\
15.55 \\
14.99 \\
14.07\end{array}$ & $\begin{array}{l}25^{*}(16) \\
26^{*} \\
27^{*}(7) \\
28^{*}(13) \\
29^{*}(9) \\
30 \\
31 \\
32^{*}(1) \\
33^{*}(3) \\
34^{*}(10) \\
35 \\
36^{*}(14) \\
37 \\
38^{*}(8 ?) \\
39 \\
40 \\
41(17) \\
42(5) \\
43 \\
44 \\
45 \\
46 \\
47\end{array}$ & $\begin{array}{l}171 \\
129 \\
147 \\
159 \\
149 \\
219 \\
107 \\
129 \\
132 \\
153 \\
209 \\
162 \\
205 \\
149 \\
128 \\
109 \\
176 \\
140 \\
104 \\
142 \\
159 \\
173 \\
150\end{array}$ & $\begin{array}{l}264 \\
264 \\
268 \\
268 \\
270 \\
273 \\
276 \\
278 \\
278 \\
281 \\
282 \\
286 \\
299 \\
302 \\
302 \\
304 \\
304 \\
307 \\
312 \\
318 \\
319 \\
328 \\
335\end{array}$ & $\begin{array}{l}14.75: \\
17.73 \\
14.08 \\
13.76 \\
13.76 \\
16.32 \\
15.92 \\
14.45 \\
15.14: \\
10.27: \\
16.70 \\
13.68 \\
15.01 \\
14.24 \\
17.59 \\
15.25 \\
14.33 \\
14.82 \\
11.61 \\
16.14 \\
16.28 \\
16.71 \\
16.20\end{array}$ & $\begin{array}{r}14.03 \\
15.38 \\
13.53 \\
12.97 \\
13.01 \\
15.70 \\
15.35 \\
13.54 \\
14.42 \\
9.52 \\
15.46 \\
12.93 \\
14.38 \\
13.56 \\
15.37 \\
14.43 \\
13.59 \\
14.05 \\
11.16 \\
15.21 \\
15.27 \\
15.49 \\
15.19\end{array}$ \\
\hline
\end{tabular}

Notes to TABLE 1

Star No. 19 is the bluest in our CM diagram and is likely a foreground star.

Star No. 24 is very red in our photometry and is very bright in the infrared POSS print, so it is very likely an M-type foreground star. 


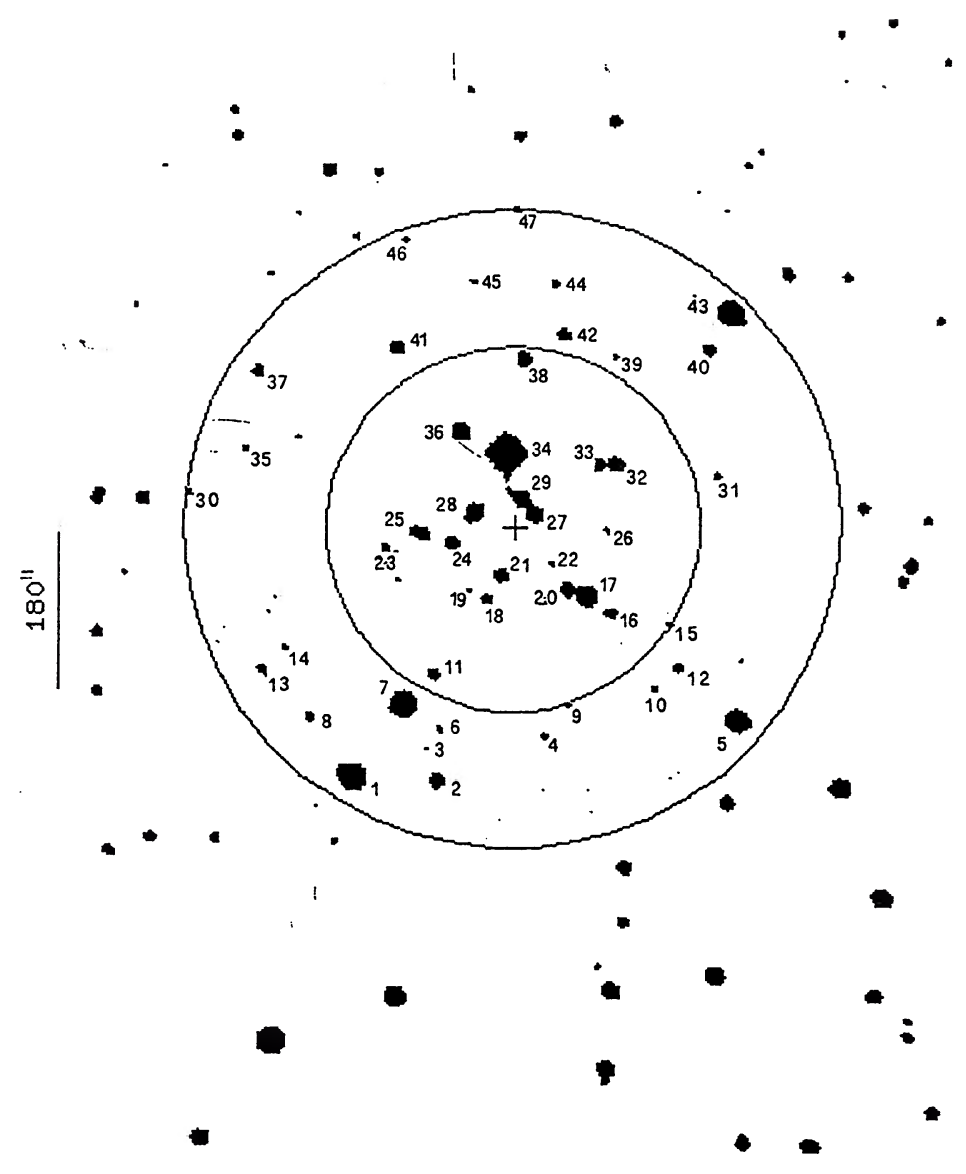

Fig. 4. Finding chart for stars brighter than $V=16.0$ in the field of NGC 433 within 70 pixel ( $=185$ arcsec) from the cluster center (indicated by a cross). The inner circle has a 40 pixel radius. North is up and east is to the left.

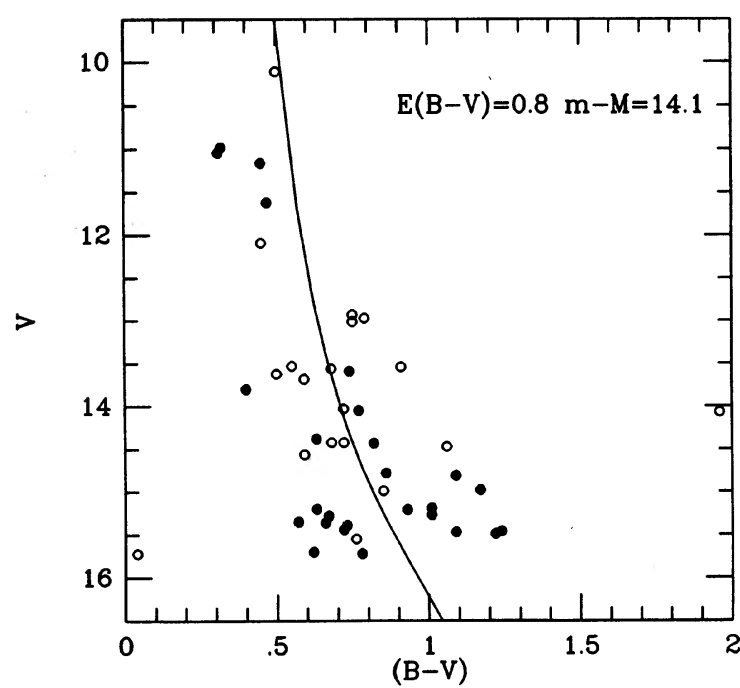

FIG. 5. Color-magnitude diagram for stars within 70 pixels $(=185$ arcsec) from the cluster center. The line is a ZAMS translated for $\mathrm{E}(B-V)=0.8$ and an apparent distance modulus of 14.1. Open circles refer to stars within 40 pixel $(=106 \mathrm{arcsec})$ from the center, filled circles to stars in the shell between 40 and 70 pixel. colors for spectral types earlier than $\mathrm{A} 0$, so we used those given by Johnson (1966), whose $(U-B)_{0}$ are almost equal to those given by Schmidt-Kaler.

\subsection{Comparison with Alter's Stars}

Our $V$ magnitudes are in good agreement with those given by Alter for 11 out of his 14 stars. Two stars (Alter's 12 and 18 ) are fainter, respectively, by 0.64 and $0.93 \mathrm{mag}$, while we could not find anything at the position of his star No. 8, which is not far from our star No. 38 and has a similar $V$ magnitude (13.46 against 13.56). Anyway, the shift in the sky is too big to be explained on the basis of proper motion, so we think that the coordinates of Alter's star No. 8 are wrong.

Alter's photographic (pg) magnitudes, transformed into $B$ magnitudes according to the formula by Arp (1957), are systematically brighter than ours: the average difference is 0.85 mag with variance 0.42 . This is likely due to systematic error in the Alter's determinations at faint magnitudes. Indeed, the diameter-magnitude relation used by Alter was calibrated over the 8-13.5 mag range: when applied to his NGC 433 observations, this relation must be extrapolated for at least 8 out of 14 stars. It can be shown that this extrapolation leads also to an underestimate of the reddening. This is the case of NGC 436, for which Huestamendia et al. (1991) 
derived $\mathrm{E}(B-V)=0.48$, while Alter (1944) gave $\mathrm{E}(B-V)=0.1$.

\section{SPECTROSCOPIC OBSERVATIONS}

It is clearly difficult, due to the large scattering of points in our color-magnitude diagram, to distinguish between cluster members and nonmembers. We decided, therefore, to perform a spectroscopic survey as deep as possible of our cluster member candidates.

Observations were performed with the $182 \mathrm{~cm}$ telescope at Cima Ekar of the Asiago Observatory, using a Boller and Chivens spectrograph with a CCD Thompson TH7882 detector (pixel size $23 \mu \mathrm{m}$ ). Brighter stars were observed at a dispersion of $85 \AA / \mathrm{mm}$, giving a resolution of $4 \AA$, in the range $\lambda \lambda 3800-5100 \AA$, while fainter stars were observed at $170 \AA / \mathrm{mm}$ and a somewhat wider slit, giving a resolution of $9 \AA$, in the range $\lambda \lambda \quad 3500-5700 \AA$. Some bright stars were observed also at the lower resolution for consistency check (see Table 2).

The classification was made by comparison with the stellar spectrophotometric library by Jacoby et al. (1984), properly degrading its higher resolution $(3 \AA)$ to that of our spectra. High resolution spectra allowed spectral types to be determined within one subtype and allowed a discrimination among luminosity classes I, III, and V. Low resolution spectra allowed the determination of the spectral type within two subtypes, leaving the distinction between luminosity classes III and V uncertain. Table 2 gives, for each star: (i) the

TABLE 2. Dispersion of the spectra $(\AA / \mathrm{mm})$, spectral type, color excess, distance (in $\mathrm{kpc}$ ) and cluster membership for the stars with spectral observations.

\begin{tabular}{cccccc}
\hline \hline Star & Dispersion & Sp. type & $\mathrm{E}(B-V)$ & Distance & Membership \\
\hline 1 & 85 & A4 V & 0.20 & 1.1 & foreground \\
2 & 170 & B8 V & 0.49 & 3.1 & background \\
5 & 85 & B5 V & 0.62 & 1.5 & foreground \\
7 & 85 & B4 V & 0.46 & 1.6 & foreground \\
$17^{*}$ & 85 & B3 III & 0.60 & 4.4 & background \\
$20^{*}$ & 170 & B6 V & $0.62:$ & $3.0:$ & member: \\
$21^{*}$ & 170 & B8 V & 0.68 & 2.3 & member \\
$25^{*}$ & 170 & B8 V & 0.79 & $2.2:$ & member: \\
$27^{*}$ & 170 & B6 V & 0.67 & 2.7 & member \\
$28^{*}$ & 170 & B6 V & 0.91 & 1.6 & member \\
$29^{*}$ & 170 & B5 V & 0.90 & 1.9 & member \\
$32^{*}$ & 170 & G5 V & 0.26 & 0.3 & foreground \\
$33^{*}$ & 170 & B9 V & $0.77:$ & 2.3 & member \\
$34^{*}$ & 85 & F6 I & $0.45:$ & $?$ & $?$ \\
$36^{*}$ & 170 & B5 V & 0.90 & 1.8 & member \\
$38^{*}$ & 170 & B6 V & 0.80 & 2.3 & member \\
41 & 170 & B8? & 0.83 & 1.7 & member \\
42 & 170 & B8 V & 0.86 & 2.1 & member \\
43 & 85 & F4 V & 0.04 & 0.3 & foreground \\
\hline \hline
\end{tabular}

Notes to TABLE 2

Asterisks refer to stars inside the 106 arcsec radius circle.

Stars No. 5, No. 17 , and No. 34 were also observed with $170 \AA / \mathrm{mm}$ dispersion; the star No. 20 is partly blended with the blue more luminous star No. 17 so its $E(B-V)$ may have been underestimated (and its distance overestimated).

Star No. 17: its spectral type is B3 III and it is less reddened than any cluster star, but its distance is about $4400 \mathrm{pc}$. This background star suggests that the extinction in the cluster direction is actually variable, maybe due to the presence of the $\mathrm{CO}$ cloud in foreground.

Star No. $34=\mathrm{BD}+59217$ : on the BD it is given $V=9.4$ in agreement with our finding. Given the large uncertainties in the absolute magnitudes of supergiants it is not possible to assign it a distance. We have checked it is not a Cepheid.
TABLE 3. Luminosity function as number of stars in the general field and in circles (whose radius is in pixels) centered on the center of NGC 433

\begin{tabular}{lccc}
\hline \hline$V$ mag. range & Field & $R=40$ & $R=70$ \\
\hline $10.5-11.0$ & 0 & 0 & 1 \\
$11.0-11.5$ & 3 & 0 & 2 \\
$11.5-12.0$ & 7 & 0 & 1 \\
$12.0-12.5$ & 11 & 1 & 1 \\
$12.5-13.0$ & 11 & 2 & 2 \\
$13.0-13.5$ & 20 & 1 & 1 \\
$13.5-14.0$ & 31 & 5 & 7 \\
$14.0-14.5$ & 39 & 5 & 8 \\
$14.5-15.0$ & 62 & 2 & 14 \\
$15.0-15.5$ & 87 & 1 & 4 \\
$15.5-16.0$ & 51 & 2 & \\
\hline \hline
\end{tabular}

spectral dispersion, (ii) the spectral type, (iii) the $\mathrm{E}(B-V)$ value computed as difference between the observed color and that given by Johnson (1966), (iv) the distance, as derived from the individual distance modulus obtained via the absolute $V$ magnitudes of Schmidt-Kaler's (1982) calibration, (v) our estimated cluster membership. Table 2 shows the existence of a clump of B stars around 2 $\mathrm{kpc}$ distance, suggesting to us to exclude as cluster members the stars at distance significantly different from this value. The average distance of "member" stars is $2.1 \pm 0.1 \mathrm{kpc}$ ( 0.1 is the standard error of the mean), with a dispersion of the single measurement of $0.3 \mathrm{kpc}$, compatible with the standard errors of our photometry and determination of the spectral type. The 106 arcsec cluster radius corresponds, therefore, to $1.1 \mathrm{pc}$. The average value of $\mathrm{E}(B-V)$ is 0.82 mag.

The distance of $2.1 \mathrm{kpc}$ is consistent with NGC 433 being associated with the $\mathrm{H}$ I clouds quoted in the introduction, while the CO cloud (at $\sim 1 \mathrm{kpc}$, Leisawitz et al. 1989) is very likely in foreground. The spectral types of the members range from B5 to B9. Assuming that the B5 stars are at the tip of the turn-off point and given a mass of $3.9 \mathscr{M}_{\odot}$ for them (Schmidt-Kaler 1982, Becker 1981) we derive as upper limit for the age $1.1 \times 10^{8} \mathrm{yr}$. Our estimate of the distance for NGC 433 implies that this cluster is 95 pc below the galactic plane, a typical value for an intermediate age open cluster (Janes \& Adler 1982).

\section{THE CLUSTER LUMINOSITY FUNCTION}

The $V$-luminosity functions (LF) for the stars contained in the external field, within 106 and within 185 arcsec from the center are given in Table 3 . The field LF decreases below $V=15.5$, due to incoming incompleteness as well as that relative to the 185 arcsec radius, while the inner circle LF shows a maximum around $V=14$.

We have identified 14 stars out of the 20 present in the inner circle: 9 as cluster members and 5 as field stars (see Tables 1 and 2). The nine ascertained members are in the narrow interval $\mathrm{B} 5 \div \mathrm{B} 9$ of spectral type [corresponding to 3.9-2.2 $\mathscr{M}_{\odot}$, from Schmidt-Kaler (1982), and Becker (1981) ]; even if all of the six stars whose membership is unknown were actual cluster members, the resulting luminosity function would be incompatible with a decreasing mass function. This serious lack of stars in the low MS suggests a truncation of the NGC 433 mass function around 
$2 \mathscr{M}_{\odot}$. This mass-function truncation is in agreement with previous studies of open cluster main sequences (see, e.g., van den Bergh \& Sher 1960). There are several possible explanations for this lack of light stars.

(i) Stars below $2 \mathscr{M}_{\odot}$ never formed because the material required for them was all locked in the building of the higher mass stars.

(ii) Low-mass stars were prevented to be born by the disruptive effects of very massive stars at the very beginning of the star formation process. We have checked whether any pulsar is known to be present in the field of the cluster (Lyne et al. 1991) but found none.

(iii) Low-mass stars are still in the process of formation. In this case, the cluster must be very young and a far infrared survey should reveal a number of cool sources. No IRAS point sources are present in the cluster area.

(iv) Low-mass stars have already been swept away from the center due to relaxation effects and only the heaviest ones remained in the central region. This hypothesis is somewhat supported by the estimate of the two-body relaxation time (based upon the numbers and masses of the stars present in the adopted cluster radius), which is of the order of one hundredth of the main sequence lifetime of the brightest cluster stars.

In conclusion, NGC 433 is an example of the populous family of relatively poor clusters [about two thirds of the whole sample reported in Lyngå (1987) are clusters with less than 30 stars], which usually consist of small clumps of stars in a restricted range of early-spectral types. These clusters seem to constitute a link between "true" bound open clusters and unbound stellar groups (see, e.g., Battinelli \& Capuzzo-Dolcetta 1991), thus suggesting, as astrophysically relevant, further studies of these type of clusters.

We thank A. Di Clemente who took some of the CCD frames at the Campo Imperatore Schmidt telecope, A. Dipaolantonio who checked for variability of the star No. 34, and the Direction of the Asiago Observatory for the allocated time.

\section{REFERENCES}

Alter, G. 1940, MNRAS, 100, 387

Alter, G. 1944, MNRAS, 104, 179

Alter, G., Ruprecht, J., \& Vanysek, V. 1970, Catalogue of Stellar Clusters and Associations, 2nd ed., edited by G. Alter, B. Balasz, and J. Ruprecht (Akademiai Kiado, Budapest)

Arp, H. 1957, ApJ, 62, 129

Battinelli, P., \& Capuzzo-Dolcetta, R. 1991, MNRAS, 249, 76

Becker, S. A. 1981, ApJS, 45, 475

Huestamendia, G., del Rio, G., \& Mermilliod, J.-C. 1991, A\&A, 87, 153

Jacoby, G. H., Hunter, D. A., \& Christian, C. A. 1984, ApJS, 56, 257

Janes, K., \& Adler, D. 1982, ApJS, 49, 425
Johnson, H. L. 1966, ARA\&A, 4, 193

Leisawitz, D., Bash, F. N., \& Thaddeus, P. 1989, ApJS, 70, 731

Leisawitz, D., \& De Geus, E. S. 1991, ApJS, 75, 835

Lyne, A. G., Manchester, R. N., \& Taylor, J. H. 1991, in Pulsar Astronomy, edited by A. G. Lyne and F. Graham-Smith (Cambridge University Press, Cambridge), p. 244

Lyngå, G. 1987, Catalogue of Open Cluster Data (C.D.S., Strasbourg)

Natali, G., \& Pedichini, F. 1990, Rev. Sci. Instrum., 61, 1839

Schmidt-Kaler, T. 1982, Landolt-Börnstein, new edition (Springer, Berlin), Vol. VIb, p. 1 .

van den Bergh, S., \& Sher, D. 1960, Publ. David Dunlap Obs., 2, 203 